\title{
OVERDETERMINATION OF THE SPEED IN RECTILINEAR MOTION OF NON-NEWTONIAN FLUIDS*
}

\section{BY J. L. ERICKSEN (Naval Research Laboratory)}

1. Introduction. The purpose of this paper is to point out that, in the case of rectilinear motion, Rivlin's theory of non-Newtonian fluids [1] yields two partial differential equations for determining a single unknown, the speed. We show that, unless the two scalars which.occur in Rivlin's theory satisfy certain definite relations, these two equations are independent. When they are independent, it is reasonable to expect only a few types of rectilinear motion to be possible. We conjecture that these exceptional motions are either rigid or such that the curves of constant speed are circles or straight lines. Stone [2] has obtained results in agreement with this for a class of ideal materials included in Rivlin's theory, these materials being very similar to those described by the theory of perfectly plastic solids.

In the case of a fluid flowing through an infinitely long cylindrical tube, to which it adheres, it would be natural to suppose that each particle could move with constant speed in a straight line parallel to the generators of the cylinder. For this to be possible, the cylinder must be a surface of constant speed for some common solution of the two equations referred to above. Suppose these are independent. Then, if our conjecture is correct, such a flow is impossible except perhaps when the cylinder is made up of portions of planes and right circular cylinders. In any event, one may reasonably expect such flows not to be possible for cylinders of arbitrary shape.

Our analysis sheds no light on the question of what type of flow replaces rectilinear motion in these tube problems when the latter is mathematically impossible and we have found no satisfactory answer to this question in the experimental literature. We are of the opinion that it need not be one of the familiar types of flow such as plug flow or turbulent flow.

It should be noted that overdetermination similar to that encountered here arises in other nonlinear theories, such as those considered in [3] and [4].

2. Equations of rectilinear motion. In Cartesian tensor notation, Rivlin's equations may be written

$$
\begin{gathered}
t_{i j, i}+\rho g_{i}=\rho\left(\partial v_{i} / \partial t+v_{i, j} v_{i}\right), \\
t_{i j}=-p \delta_{i j}+\mathcal{F}_{1}\left(I_{2}, I_{3}\right) d_{i j}+\mathcal{F}_{2}\left(I_{2}, I_{3}\right) d_{i k} d_{k i}, \\
v_{i, i}=0,
\end{gathered}
$$

where $t_{i j}$ is the stress tensor, $g_{i}$ the body force per unit mass, $v_{i}$ the velocity vector, $d_{i j}=1 / 2\left(v_{i, i}+v_{i, i}\right)$ the rate of deformation tensor, $2 I_{2}=-d_{i j} d_{i j}, I_{3}=\operatorname{det} d_{i j}, p$ is an arbitrary hydrostatic pressure, and $\rho$ is the density, assumed constant. The $F$ 's are essentially arbitrary functions of the indicated arguments, to be determined by experiment.

If each particle moves with constant speed in a straight line parallel to the $x_{3}$-axis, then $v_{1}=v_{2}=0, \partial v_{3} / \partial t+v_{3.3} v_{3}=0$. If we further require (3) to hold, we obtain $v_{3,3}=\partial v_{3} / \partial t=0$, from which $v_{3}=2 f\left(x_{1}, x_{2}\right)$, the factor 2 heing introduced to simplify

*Received Sept. 12, 1955. 
expressions to follow. Then $I_{2}=-f_{. i} f_{, i}, I_{3}=0$, and, from (2),

$$
\left\|t_{i j}\right\|=-p\left\|\delta_{i i}\right\|+g_{1}\left\|\begin{array}{ccc}
0 & 0 & f_{.1} \\
0 & 0 & f_{.2} \\
f_{, 1} & f_{.2} & 0
\end{array}\right\|+\varsigma_{2}\left\|\begin{array}{ccc}
f_{.1}^{2} & f_{.1} f_{.2} & 0 \\
f_{.1} f_{.2} & f_{.2}^{2} & 0 \\
0 & 0 & f_{. i} f_{, i}
\end{array}\right\|
$$

where $G_{i}=\mathcal{F}_{i}\left(I_{2}, 0\right)$. We assume the body force conservative, $g_{i}=\varphi_{. i}$, and henceforth restrict indices to values 1 and 2 . Using (1) and (4), we then obtain

$$
P_{, i}=\left(\mathcal{G}_{2} f_{, i} f_{, i}\right)_{, i}, \quad P_{, 3}=\left(\mathcal{G}_{1} f_{. i}\right)_{, i},
$$

where $P=p-\rho \varphi$ is an arbitrary hydrostatic pressure. From (5), $P_{. \text {i } 3}=P_{.33}=0$, so $P=a x_{3}+Q\left(x_{1}, x_{2}\right)$, where $a$ is a constant. Then

$$
Q_{, i}=\left(\mathcal{G}_{2} f_{, i} f_{, i}\right)_{, i}=\left(\mathcal{G}_{2} f_{, i}\right)_{, i} f_{, i}-\frac{1}{2} \mathcal{G}_{2} I_{2, i},
$$

so that the gradient of $R=Q+\frac{1}{2} \int \mathcal{G}_{2} d I_{2}$ is parallel to that of $f$. Hence $R=R(f)$ and we have

$$
\begin{aligned}
d R / d f & =\left(\mathcal{S}_{2} f_{, i}\right)_{, i}=\mathcal{S}_{2}^{\prime} I_{2, i} f_{, i}+\mathcal{S}_{2} f_{, i j}, \\
a & =\left(\mathcal{G}_{1} f_{, j}\right)_{, j}=\mathcal{G}_{1}^{\prime} I_{2, i} f_{, i}+\mathcal{G}_{1} f_{, i j},
\end{aligned}
$$

where primes denote differentiation with respect to $I_{2}$. Equation (7) is equivalent to the integrability conditions $Q_{.12}=Q_{.21}$ and either of these, with (8), gives two differential equations for determining $f$.

3. Dependency conditions. Suppose that, in some open interval $g$ of values of $I_{2}$, we have

$$
\left(\mathcal{G}_{1} \mathcal{G}_{2}^{\prime}-\mathcal{G}_{1}^{\prime} \mathrm{G}_{2}\right)\left(\mathrm{G}_{1}+2 I_{2} \mathcal{G}_{1}^{\prime}\right) \neq 0 .
$$

Suppose further that $\mathcal{G}_{1}$ is an analytic function of $I_{2}$ in $g$. Under these conditions, we will show that, for every $a$, there exist solutions of (8) such that (7) is not satisfied for any choice of $R(f)$. To establish this, we consider a Cauchy problem for (8). Let $C$ be an analytic curve, given parametrically in terms of its arc length $s$ by $x_{i}=x_{i}(s)$, so that $d x_{i} / d s d x_{i} / d s=1$. This curve may be chosen arbitrarily, subject to the restriction that its curvature not be constant. Let $I_{2}^{\prime}<0$ be any value of $I_{2}$ in $g$ and let $b=\left(-I_{2}^{\prime}\right)^{1 / 2}$. A Cauchy problem is set by specifying values of $f$ and $f_{. i}$ on $C$ such that the compatibility condition $d f / d s=f_{. i} d x_{i} / d s$ is satisfied. In particular, we may set

$$
f=0, \quad f_{, 1}=b d x_{2} / d s, \quad f_{, 2}=-b d x_{2} / d s
$$

on $C$. Differentiating with respect to $s$, we obtain, on $C$,

$$
\begin{aligned}
& f_{.1 i} d x_{i} / d s=d f_{, 1} / d s=b d^{2} x_{2} / d s^{2}=b K d x_{1} / d s, \\
& f_{, 2 i} d x_{i} / d s=d f_{, 2} / d s=-b d^{2} x_{1} / d s^{2}=b K d x_{2} / d s,
\end{aligned}
$$

where $K$ is the curvature of $C$, given by $K^{2}=d^{2} x_{i} / d s^{2} d^{2} x_{i} / d s^{2}$. Now (8) and (11) provide three linear equations for determining the three unknowns $f_{. i j}$ as functions of $s$. Using the facts that $I_{2, i} f_{, i}=-2 f_{, i} f_{, i} f_{, i}$ and that (10) holds, we find that the determinant 
$\Delta$ of the coefficients in these equations is given by

$$
\begin{aligned}
\Delta & =\left(d x_{1} / d s\right)^{2}\left[\mathcal{G}_{1}-2 f_{.2}^{2} \mathcal{G}_{1}^{\prime}\right]+\left(d x_{2} / d s\right)^{2}\left[\mathcal{G}_{1}-2 f_{.2}^{2} \mathcal{G}_{1}^{\prime}\right]+4\left(d x_{1} / d s\right)\left(d x_{2} / d s\right) f_{.1} f_{.2} \mathcal{G}_{1}^{\prime} \\
& =\mathcal{S}_{1}-2 b^{2} \mathcal{G}_{1}^{\prime} .
\end{aligned}
$$

On $C, I_{2}=-b^{2}$, so (9) implies that $\Delta \neq 0$. Hence the $f_{, i j}$ are determined uniquely. Similarly, all higher derivatives of $f$ can be determined on $C$, so we can determine a power series expansion of $f$ about a point $P$ on $C$. The fact that, in some circle of nonzero radius about $P$, this power series converges to a solution of (8) can be inferred from known results in the theory of partial differential equations. For example, one can refer all equations to an orthogonal curvilinear coordinate system in which $C$ is a coordinate curve, then make use of the Cauchy-Kowalewsky existence theorem [5, Sec. 94]. It is relatively simple to show that the solution thus obtained cannot satisfy (7). On $C$, $f=0$ and $I_{2}$ is constant, so that $d R / d f, \mathcal{S}_{1}, \mathcal{G}_{1}^{\prime}, \mathcal{S}_{2}$ and $\mathcal{G}_{2}^{\prime}$ must be constant. If (7) and (8) were both satisfied, we could, since $\mathcal{S}_{1} \mathcal{S}_{2}^{\prime}-\mathcal{G}_{2} \mathcal{S}_{1}^{\prime} \neq 0$, solve (7) and (8) for $f_{, i i}$ and $I_{2, i} f_{, i}$. These quantities would then be constant on $C$. On the other hand, on $C$,

$$
\begin{aligned}
& I_{2, i} f_{, i}=-2 f_{, i} f_{. i} f_{. i}=- 2 b^{2} f_{, 11}\left(d x_{2} / d s\right)^{2}-2 b^{2} f_{, 22}\left(d x_{1} / d s\right)^{2} \\
& \quad+4 b^{2} f_{, 12} d x_{1} / d s d x_{2} / d s=-2 b^{2} f_{, i i}+2 b^{2} f_{, i i} d x_{i} / d s d x_{i} / d s \\
&=-2 b^{2} f_{, i i}+2 b^{2} d f_{, i} / d s d x_{i} / d s=-2 b^{2} f_{, i i}+2 b^{2} K,
\end{aligned}
$$

where we have used (10), (11) and $d x_{i} / d s d x_{i} / d s=1$. Thus, if $I_{2, i} f_{, i}$ and $f_{. i i}$ were both constant on $C$, we would have $b^{2} K$ constant, which contradicts the assumptions $I_{2}^{\prime}<0$ and $K \neq$ constant made earlier. Thus $f$ cannot satisfy (7).

Excluding the pathological case where $S_{1}$ is not analytic in any interval where (9) holds, we have left to consider what occurs if the left member of (9) vanishes for all $I_{2}$. We assume $S_{1} \neq 0$ which will be the case if, for example, the inequalities discussed in [6] are satisfied. If $\mathcal{S}_{1} \mathcal{G}_{2}^{\prime} \equiv \mathcal{G}_{2} \mathcal{G}_{1}^{\prime}$, we then have $\mathcal{G}_{2}=\alpha \mathcal{G}_{1}$, where $\alpha$ is a constant, or, in terms of previous notation,

$$
\mathcal{F}_{2}\left(I_{2}, 0\right)=\alpha \mathcal{F}_{1}\left(I_{2}, 0\right) .
$$

Whenever (12) holds, every solution of (8) satisfies (7) with $d R / d f=a \alpha$, so $f$ is not overdetermined. It then follows that a function $f$ satisfying the basic equations with $\mathcal{F}_{2}=0$ will satisfy them with $\mathcal{F}_{2}$ given by (12) for any $\alpha$. Oldroyd has discussed several methods of obtaining solutions for rectilinear motion of materials for which $\mathcal{F}_{2} \equiv 0$ [7], [8], [9]. It is perhaps worth mentioning that, if (12) holds with $\alpha \neq 0$ for all motions such that $I_{3}=0$, there will exist motions for which the inequalities discussed in [6] are not satisfied. If $\mathcal{S}_{1}+2 I_{2} \mathcal{G}_{1}^{\prime} \equiv 0, \mathcal{S}_{1}=k\left(-I_{2}\right)^{-1 / 2}$, where $k$ is a non-zero constant. In this case, the curves $f=$ constant must be straight lines or circles, as has been shown by Stone [2].

\section{References}

[1] R. S. Rivlin, Hydrodynamics of non-Newtonian fluids, Nature 160, 611-613 (1947)

[2] D. E. Stone, On nonexistence of rectilinear motion in plastic solids and non-Newtonian fluids, forthcoming

[3] W. Noll, On the continuity of the solid and fluid states, J. Rational Mech. Anal. 4, 3-81 (1955)

[4] R. S. Rivlin and J. L. Ericksen, Stress-deformation relations for isotropic materials, J. Rational Mech. Anal. 4, 323-425 (1955) 
[5] E. Goursat, $A$ course in mathematical analysis, translated by E. R. Hedrick and O. Dunkel, Ginn and Co., Boston, 1917, vol. 2

[6] M. Baker and J. L. Ericksen, Inequalities restricting the form of the stress-deformation relations for isotropic elastic solids and Reiner-Rivlin fluids, J. Wash. Acad. Sci. 44, 33-35 (1954)

[7] J. G. Oldroyd, Rectilinear plastic flow of a Bingham solid. III. A more general discussion of steady flow, Proc. Cambr. Phil. Soc. 44, 200-213 (1948)

[8] J. G. Oldroyd, Rectilinear flow of non-Bingham plastic solids and non-Newtonian viscous liquids. I, Proc. Cambr. Phil. Soc. 45, 595-611 (1949)

[9] J. G. Oldroyd, Rectilinear flow of non-Bingham plastic solids and non-Newtonian viscons liquids. II, Proc. Cambr. Phil. Soc. 47, 575-584 (1951)

\section{VECTOR FIELDS ASSOCIATED WITH PLANE PLASTICITY*}

By D. R. BLAND (King's College, London)

Synopsis. A symmetric tensor can be associated with a vector field by means of Eq. (1). It is found that, for the stress field in plane plasticity, associated vector fields can always be found. For certain vector fields, associated stress fields exist. Examples are given.

Transformation between stress and vector. The most general way in two dimensions that the symmetric stress tensor $\sigma_{i i}$ can be associated with a vector field $v_{i}$, when no derivatives or integrals of $v_{i}$ are included, is

$$
\sigma_{i j}=M \delta_{i j}+N v_{i} v_{i}
$$

where $M$ and $N$ are functions of $v_{k}^{2}$. The stress tensor satisfies $\sigma_{i i, j}=0$. Hence, from (1),

$$
2 M^{\prime} v_{k} v_{k, i}+2 N^{\prime} v_{k} v_{k, j} v_{i} v_{i}+N v_{i, j} v_{i}+N v_{i} v_{i, i}=0 .
$$

Resolving the vector equation (2) parallel and normal to $v_{i}$,

$$
\left(2 M^{\prime}+2 N^{\prime} v_{k}^{2}+N\right) v_{i} v_{i} v_{i, i}+N v_{i}^{2} v_{i, j}=0
$$

and **

$$
\epsilon_{i l} v_{i} v_{l}\left(2 M^{\prime} v_{i, i}+N v_{i, i}\right)=0 .
$$

The invariants of the stress tensor can be found in terms of $M, N$ and $v_{k}^{2}$,

$$
I_{1}=\sigma_{i i}=2 M+N v_{k}^{2}
$$

and

$$
I_{2}=\sigma_{i j} \sigma_{i j}=2 M^{2}+2 M N v_{k}^{2}+N^{2}\left(v_{k}^{2}\right)^{2} .
$$

The stress tensor for a solid in the plastic state satisfies a criterion of the form

$$
f\left(I_{1}, I_{2}\right)=0 \text {. }
$$

Substituting from (5) and (6),

$$
g\left(M, N, v_{k}^{2}\right)=0,
$$

${ }^{*}$ Received Sept. 28, 1955; revised manuscript received November 18, 1955.

${ }^{* *} \epsilon_{i j}$ is the tensor $\epsilon_{11}=\epsilon_{22}=0, \epsilon_{12}=1, \epsilon_{21}=-1$. 